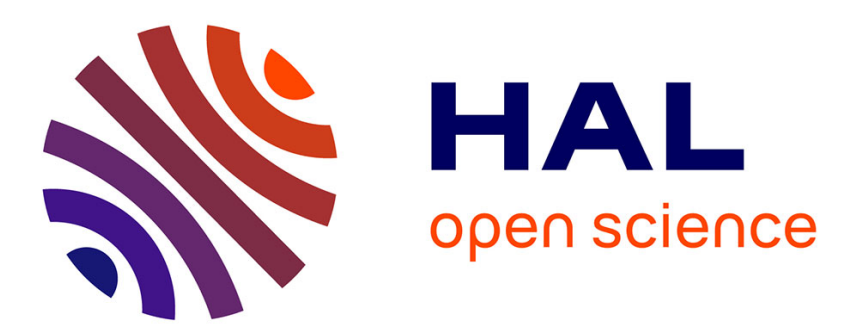

\title{
Force-Position Photo-Robotic Approach for the High-Accurate Micro-Assembly of Photonic Devices
}

\author{
Houari Bettahar, Cédric Clevy, Nadège Courjal, Philippe Lutz
}

\section{To cite this version:}

Houari Bettahar, Cédric Clevy, Nadège Courjal, Philippe Lutz. Force-Position Photo-Robotic Approach for the High-Accurate Micro-Assembly of Photonic Devices. IEEE Robotics and Automation Letters, 2020, 5 (4), pp.6396 - 6402. 10.1109/LRA.2020.3014634 . hal-03420172

\section{HAL Id: hal-03420172 \\ https://hal.science/hal-03420172}

Submitted on 27 Jan 2022

HAL is a multi-disciplinary open access archive for the deposit and dissemination of scientific research documents, whether they are published or not. The documents may come from teaching and research institutions in France or abroad, or from public or private research centers.
L'archive ouverte pluridisciplinaire HAL, est destinée au dépôt et à la diffusion de documents scientifiques de niveau recherche, publiés ou non, émanant des établissements d'enseignement et de recherche français ou étrangers, des laboratoires publics ou privés. 


\title{
Force-Position Photo-Robotic Approach for the High-Accurate Micro-Assembly of Photonic Devices.
}

\author{
Houari Bettahar, Cédric Clévy, Nadège Courjal and Philippe Lutz
}

\begin{abstract}
Recently, micro-assembly of individual photonic elements has been highly attracting to provide new optical functionalities and products with high performances. This approach requires position control in free space and contact detection in constrained space. In both cases, getting accurate measurements remains a key lock. For this sake, the FP (FabryPerot) interferences happening during the active alignment of optical elements is exploited. This 1D displacement measurement is combined with multi-DoF (Degrees-of-Freedom) robotic motions and a compliant structure along a photo-robotic approach to provide high accurate estimation of both multiDoF position and contact forces during the assembly process. This approach has been applied to the assembly of an optical lamella with respect to a fiber. An original contact detection algorithm has been proposed, which relies on correlations between the interference figure and the expected irradiance deduced from the nano-positioner internal sensors. This contact detection enables to estimate the position of the lamella with respect to the fiber along the optical axis and thus control the UV-adhesive thickness required to achieve high optical performances after assembly. Experimental investigations using this new approach shows that a positioning accuracy of $27.6 \mathrm{~nm}$ and $2.1 \mathrm{~m}^{\circ}$ (standard deviation values) are reached for positions and orientations respectively. The contact detection algorithm has also been studied experimentally and results shows that the contact position can be detected with a maximum error of $80 \mathrm{~nm}$, which provides a very high interest for many photonic applications.
\end{abstract}

Index Terms-Micro-robotics, Nano-positioning, Accuracy, Photo-robotic, Photonics, Fabry-Perot interferences.

\section{INTRODUCTION}

Achieving fast and automated 3D complex tasks of micromanipulation and micro-assembly with very high accuracy is strongly desired in many application fields, such as advanced integrated optics manufacturing (to achieve commercial success with reliable and cost-effective assembly, and packaging technologies) [1] [2]. Many emerging industrial on-demand applications made the field of integrated optics particularly challenging because of their always increasing functions and complexity. Indeed, these applications require the integration of various building components coming from different technologies in order to achieve a wide range of microsystem types [3] [4]. The possibility to achieve hybrid nano-photonic components by assembly provides very original solutions to overcome the monolithical integration difficulties and to achieve 3D multi-functional optical devices fabricated with different technologies [4]. Robotic micro-assembly also enables to automate, to optimize the optical performances through closed loop control and to adapt every assembly to the heterogeneities of fabrication processes [5].

Positioning and assembly for integrated optics industry is generally done manually based on passive and active positioning for many applications [6]. In passive positioning [7], operators may use geometrical devices such as V-grooves, or visual systems such as a microscope or a binocular to provide visual geometrical information which could be used for positioning [8] [9]. However, very complex procedures must be established in passive positioning to guarantee optical performances. For this reason, the active approach is mainly used for integrated optics industry and mostly in a manual or teleoperated way [10] [11]. Micro and nano-positioning systems are used to adjust the position of the optical component in order to obtain the maximum optical irradiance. This manipulation requires dexterity and skills on the part of the operator and a lot of precision and attention which means that it is a tiring task for the operator. Consequently, the performances and quality of the assembled products are strongly operator-dependent. Moreover, optical components are fragile and easily breakable. They typically break at the micro-Newton $(\mu \mathrm{N})$ force range, a range that cannot be felt by a human (then by the operator) assembling these optical components with tweezers and microscopes. It is also, not reliably measurable with the existing force sensors during assembly. As a result, it is extremely difficult to manipulate effectively and flawlessly optical components for assembly at that scale. This situation decreases overall yields, drives up the assembled optical structure costs. and prevent from scaling up.

Furthermore, closed loop control approaches typically used in robotics opens the possibility to control the relative position between two components in a referenced way. Nevertheless, at the microscale, micro and nanopositioning robots introduce geometrical errors and integrating sensors able to estimate contact forces in the $\mathrm{mN}$ range together with relative position is still an open issue [12] [13].

In this scope, a photo-robotic approach was proposed in the previous works [14] [15]. It has especially been applied to align an optical lamella with respect to an optical fiber with high accuracy in free space and without any contact between the two components. This approach uses a 1-D interferometric measurement principle based on FP interferences associated to a robot motion in order to achieve identification of the extrinsic geometric parameters. The working principle of this approach was presented and validated experimentally. It demonstrated its interest and high performances for different cases: identification of 2 parameters [14], identification of 4 parameters [15] and identification of all extrinsic parameters of the robot [16].

These works was indeed based on an "position"-based photo-robotic approach and demonstrated the capability to achieve the relative positioning of two optical components. 
But, achieving the full assembly process require to consider the contacts between the components notably to control the UV-adhesive thickness required to maintain high optical performances after completing the assembly process. For these purposes, the contribution of the paper especially aims at proposing an algorithm to detect the contact (i.e. the relative distance) between the two optical components. The proposed approach especially synergize both position and force necessary to complete the whole assembly process. This approach is based on the correlation between FP cavity length and the interference figure and thus conduct to an original "force-position"-based photo-robotic approach.

The paper is organized as follows. Section II recalls the FP interferometry working principle. Section III illustrates the correlation between the robotic motions and the FP interference figure and presents the photo-robotic approach. Section IV presents the "force-position" photo-robotic approach for multi-DOF nano-positioning. Section IV investigates the strategy to maximize optical performances. Section $\mathrm{V}$ investigates contact detection based on FP interferometry. Section VI concludes the paper.

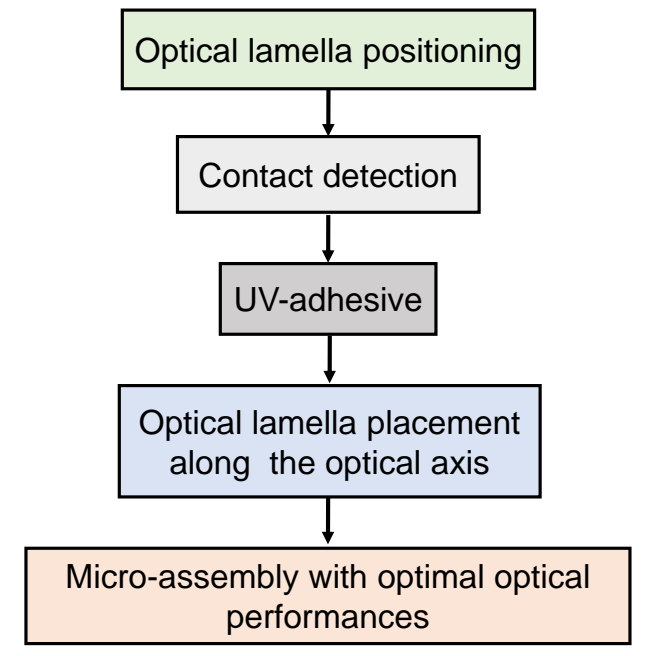

Fig. 1. The proposed micro-assembly task flowchart.

\section{FABRY-PEROT INTERFEROMETRY}

Interferometric sensing is attracting due to its high resolution, accuracy, wide measuring range and bandwidth. Active alignment induces the FP interferometry phenomenon resulted by the multiple beam interferences that arises when light passes through a cavity bounded by two reflective surfaces as shown in Figure 2. A generic optical system is chosen as a case-study. This generic optical system represents an optical fiber and an optical lamella, as depicted in Figure 2. In our case, the involved surfaces are the fiber extremity $\left(R_{1}\right)$ in one hand and the lamella surface $\left(R_{2}\right)$ in the other hand.

Based on the FP interferometry principle, when the reflective surface $\left(R_{2}\right)$ moves along the optical axis, the FP cavity length $(L)$ changes and modifies the reflected light signal accordingly. Therefore, there is a correlation between

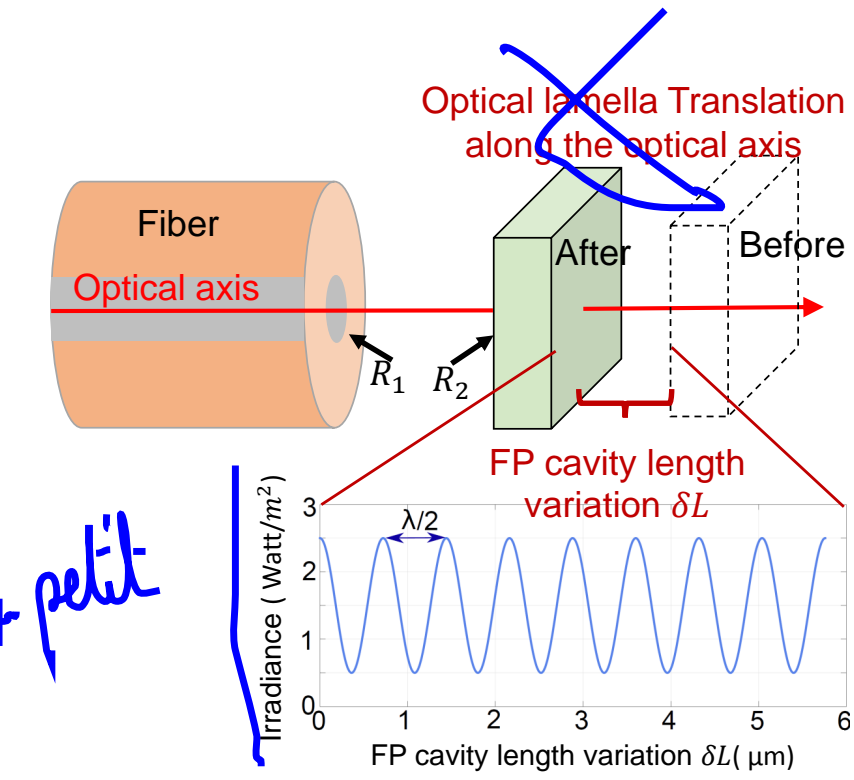

Fig. 2. Schematic diagram of the case-study optical system (fiber, optical lamella).A translation of the optical lamella along the optical axis produces simultaneously a sinusoidal Reflected irradiance.

the FP cavity length $L$ and the interference figure, defined as follows:

$$
\delta L=\frac{\lambda}{2} \cdot p \quad \text { with } \quad p \in N
$$

The periodicity of the irradiance signal depends directly on the wavelength of the laser signal $(\lambda=1560 \mathrm{~nm}) . p$ is a natural number, the distance between two resonances (see Figure 2, i.e. the distance variation between two peaks $(\delta L)$ is a multiple of half wavelength $\lambda$ of the laser irradiance.

Active positioning and assembly of optical components give the opportunity to measure a highly accurate 1-D relative position along the optical axis with very high resolution based on 1-D FP interference.

\section{PHOTO-ROBOTIC POSITIONING APPROACH FOR HIGHLY ACCURATE NANO-POSITIONING}

Conventional active alignment techniques is done manually by an operator. The operator moves the optical lamella with respect to the fiber, in order to maximize the optical irradiance, by applying a an non-referenced movements via the nano-positioner. As a results, it leads to local maximum optical irradiance. Moreover, it is time consumption especially for multi-DOF alignment process to reach maximum irradiance, since there is no model relating robotic control variables with the optical irradiance.

For this sake, the proposed photo-robotic approach of our previous paper [15] is used, to achieve highly accurate multiDOF positioning. The photo-robotic approach uses a 6-DOF serial nano-positioning system to hold the optical lamella and align it to a fixed fiber as shown in Figure 3 The positioning system is controlled via the computer using Matlab/Simulink platform. The interfered reflected light is measured using the photo-detector (optical irradiance), where the fibered circulator is used to separate the reflected interfered light from the incident light. The measured reflected light 


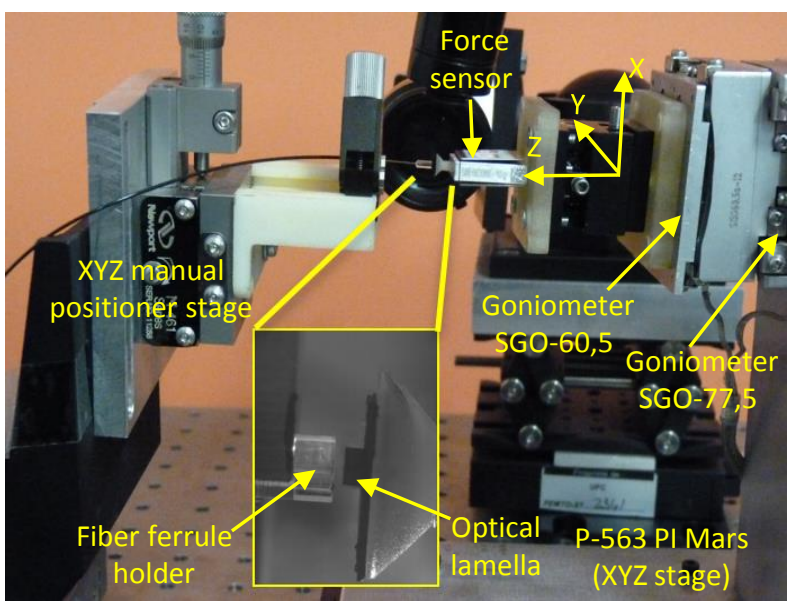

Fig. 3. Experimental setup: a 6 DOF nano-positioner used to move a photonic component in front of a fiber.

TABLE I

THE ASSIGNED REFERENCE FRAMES DEFINITIONS.

\begin{tabular}{|c|c|}
\hline Reference frame & Definition \\
\hline$R_{p}\left(O_{p} X_{p} Y_{p} Z_{p}\right)$ & $\begin{array}{l}\text { is assigned to the positioning system, the } \\
\text { origin } O_{p} \text { is assigned to its real rotational } \\
\text { center of the positioning system, the } \overrightarrow{O_{p} X_{p}} \text {, } \\
\overrightarrow{O_{p} Y_{p}} \text { and } \overrightarrow{O_{p} Z_{p}} \text { are the expected motion } \\
\text { directions of the positioning system. }\end{array}$ \\
\hline$R_{l}\left(O_{l} X_{l} Y_{l} Z_{l}\right)$ & $\begin{array}{l}\text { is assigned to the optical lamella, its origin } \\
\text { is assigned to the intersection point between } \\
\text { the optical axis and the lamella plane. The } \\
\text { axis } \overrightarrow{O_{l} Z_{l}} \text { is perpendicular to the optical } \\
\text { lamella plane and it is directed toward the } \\
\text { fiber. The axes } \overrightarrow{O_{l} Y_{l}} \text { and } \overrightarrow{O_{l} X_{l}} \text { are perpen- } \\
\text { dicular to each other and they are on the } \\
\text { lamella plane. }\end{array}$ \\
\hline$R_{f}\left(O_{f} X_{f} Y_{f} Z_{f}\right)$ & $\begin{array}{l}\text { is assigned to the fiber, its origin } O_{f} \text { is } \\
\text { assigned to the intersection point between } \\
\text { the optical axis and the fiber plane. The } \\
\text { axis } \overrightarrow{O_{f} Z_{f}} \text { is perpendicular to the optical } \\
\text { lamella plane and it is carried by the optical } \\
\text { axis and it is directed toward the optical } \\
\text { lamella. The axes } \overrightarrow{O_{f} Y_{f}} \text { and } \overrightarrow{O_{f} X_{f}} \text { are } \\
\text { perpendicular to each other and they are on } \\
\text { the fiber plane. }\end{array}$ \\
\hline
\end{tabular}

by the photo-detector is also acquired using an acquisition card interfaced with Matlab/Simulink platform. The optical lamella is adjusted using the nano-positioning system in order to search the maximum optical irradiance. As a result, several parameters influencing the interference figure need to control the optical response through controlling the nanopositioning.

Three reference frames are defined, one is assigned to the positioning system, the second is assigned to the optical lamella and the last is assigned to the fiber as shown in Figure 5. The transformation between each two reference frames is achieved by using transformation matrices including the geometric parameters between frames [16]. The definition of each reference frame is in Table I.
Figure 5 summarizes schematically the whole diagram of the photo-robotic approach. The multi-DOF nano-positioner motion allows to position the optical lamella with respect to the fiber. A 1-D relative distance measurement is achieved using the interferometry figure for each nanopositioner motion. Different optical lamella geometric parameters $\left(\beta_{i}, \theta_{i}, \beta_{l}, \theta_{l}\right)$ can be identified using the 1-D relative distance measurements and the multi-DOF nanopositioner motion. $\beta_{i}$ is the angle between the plan of the optical lamella and the axis parallel to $Y_{f}$ axis passing by the origin $O_{l}$, in the $\left(Y_{f} O_{f} Z_{f}\right)$ plan. $\beta_{l}$ is the angle between the real axis $X_{p}$ and the axis parallel to $Y_{f}$ axis passing by the origin $O_{l}$ in the $\left(Y_{f} O_{f} Z_{f}\right)$ plan (see Figure 4). $\theta_{i}$ is the angle between the surface of the optical lamella and the axis parallel to $X_{f}$ axis passing by the origin $O_{l}$, in the $\left(X_{f} O_{f} Z_{f}\right)$ plan. $\theta_{l}$ is the angle between the $Y_{p}$-axis (real displacement axis) and the axis parallel to $X_{f}$ axis passing through the origin $O_{l}$, in the $\left(X_{f} O_{f} Z_{f}\right)$ plan.

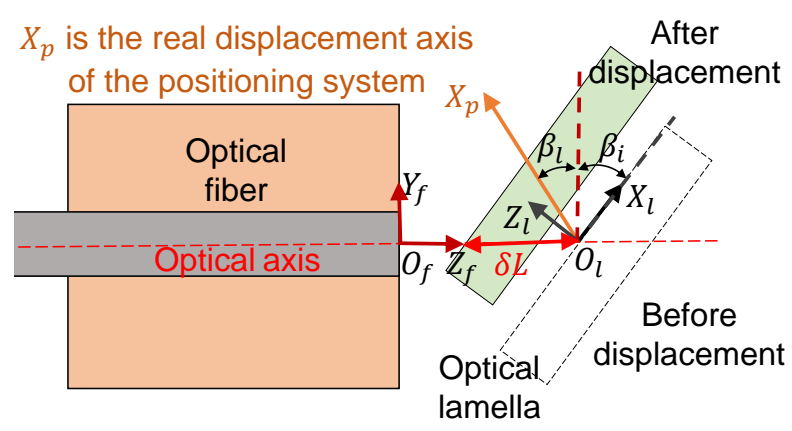

Fig. 4. Real displacement is along $X_{p}$ and it is coupled with perpendicularity default angle $\beta_{i}$, frames referencing angle $\beta_{l}$ and $\delta L$ displacement along the optical axis.

Finally, a photo-robotic model is used to calculate the control angles for the nano-positioner $\left(\beta_{p}, \theta_{p}\right)$ to be applied. The uncertainty distribution of the identified relative misalignment angles $\beta_{i}$ for each given nano-positioner angle was investigated as well. We obtained rotational measurements with an uncertainty based on standard deviation is 2.1 $\mathrm{m}^{\circ}$, and the translational measurements with an uncertainty based on standard deviation is $27.6 \mathrm{~nm}$.

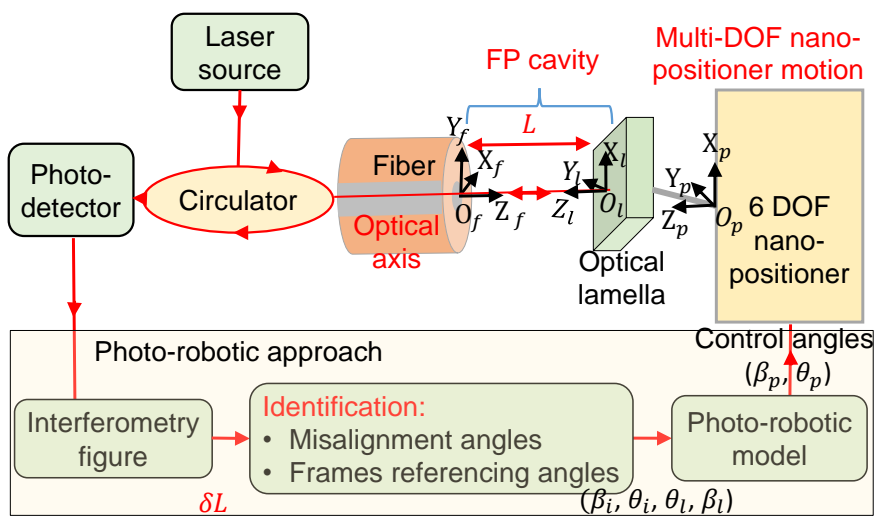

Fig. 5. The photo-robotic approach experimental implementation. 


\section{MAXIMIZATION OF OPTICAL IRRADIANCE}

In order to understand the irradiance maximization issue, we suppose that, a robotic referenced displacement without relative misalignment angles is applied along $Y_{f}$ or $X_{f}$ axis. The obtained optical irradiance is expected to be constant, but the maximum optical irradiance is not guaranteed.

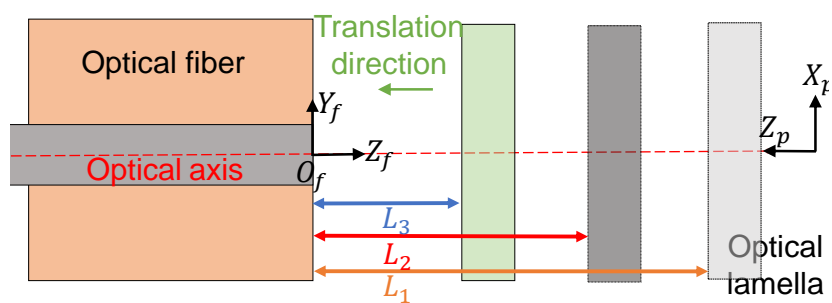

Fig. 6. Translation along the optical axis ( $Z_{f}$-axis) toward the fiber.

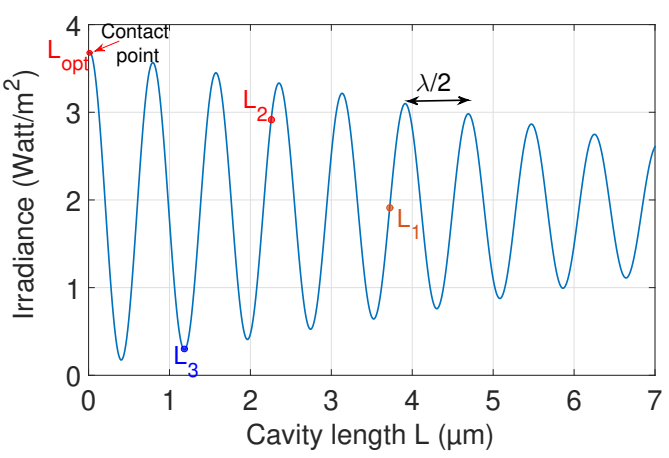

Fig. 7. Correlation between the FP cavity length $L$ and a simulated FP interference for translation along the optical axis toward the fiber.

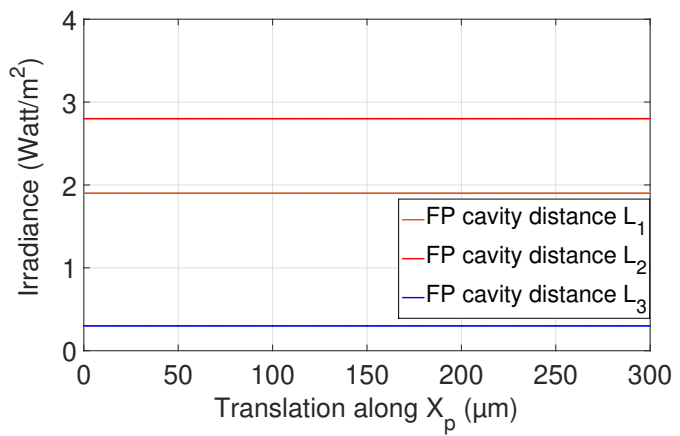

Fig. 8. The optical irradiance simulation corresponding to different FP cavity length when translating along $X_{p}$-axis.

When the optical lamella is translated along the optical axis, toward the fiber without misalignment angles as shown in Figure 6, the corresponding optical irradiance varies increasingly as shown in Figure 7. The closest the optical lamella to the fiber is, the highest oscillation amplitudes (peaks) of the optical irradiance are. If the optical lamella is placed on different FP cavity lengths, from the farthest to the closest $\left(L_{1}, L_{2}, L_{3}\right)$ respectively as shown in Figure 6 . the corresponding optical irradiance to the closest FP cavity length $L_{3}$ is the lowest compared to those corresponding to the cavity length $L_{1}, L_{2}$ even if it is the closest to the optical fiber. The optical irradiance which corresponds to the FP cavity length $L_{2}$ is higher than the optical irradiance which corresponds to the FP cavity length $L_{3}$ even it is farther to the optical fiber than FP cavity length $L_{3}$ (see Figure 8). Therefore, the optical irradiance depends on if the induced cavity length corresponds to a maximum peak or not. The correlation between FP cavity length and the interference figure is essential to guarantee the maximum optical irradiance. However, in order to achieve the optimal maximum irradiance, the first closest peak before contact has to be attained. Contact detection is discussed in the next section.

\section{Contact Detection BASEd on FABRy-PERot INTERFEROMETRY}

Micro-assembly of optical components requires position control for both, free movements and constrained movements i.e. when the contacts occur. Micro-assembly requires potentially contact detection in constrained space. The correlation between FP cavity length and the interference figure is essential to guarantee the maximum optical irradiance during assembly. In order to achieve the maximum optical irradiance, the first closest peak before contact has to be attained. Therefore, in order to reach maximum optical irradiance, contact detection between the optical lamella and the fiber is necessary, Once the contact is identified, the first maximum peak before contact can be reached.

The contact detection can be achieved by integrating a compliant structure between the lamella and the 6-DOF positioner. In this work we propose to perform contact detection and force estimation based on FP interferometry measure. In order to validate the proposed approach a reference force sensor is integrated to the experimental setup. In this way, the results obtained from force estimation based on FP interferometry measurements are compared to those obtained from the force sensor.

To study the proposed principle, the experimental setup has been modified as schematically shown in Figure 9. The real experimental setup can be seen on Figure 3

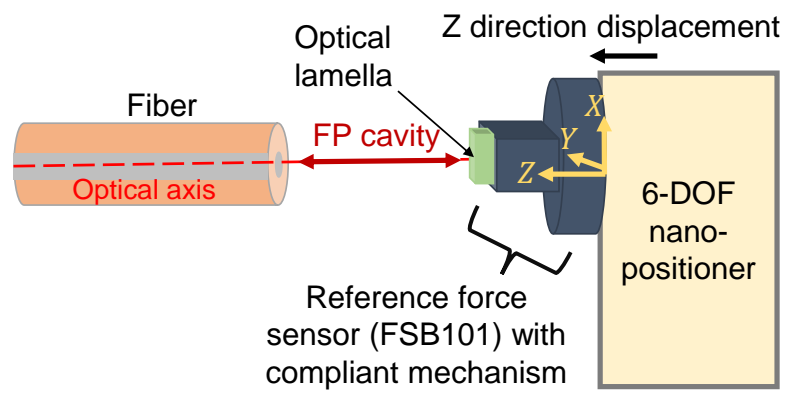

Fig. 9. Experimental set-up scheme for force estimation and contact detection .

The first step in the validation process is to investigate the repeatability of the force sensor placed at the end-effector of the 6-DOF nano-positioner. For this sake, a forwardbackward motion on the Z-axis is done, where the contact of the optical lamella with the fiber is reached for each forward motion and the displacement continues to estimate the incurred force. Since we are interested in the contact detection, the repeatability of the detected force signal for forward displacement is provided in Figure 10. 


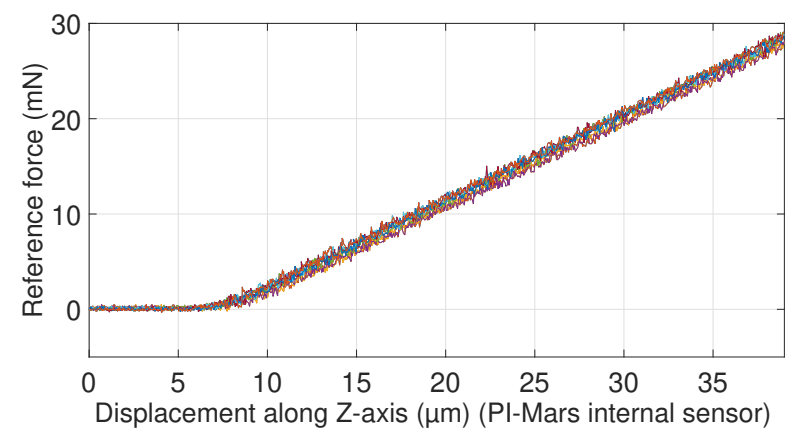

Fig. 10. The corresponding experimental sensed force for 7 forth motion along the Z-axis.

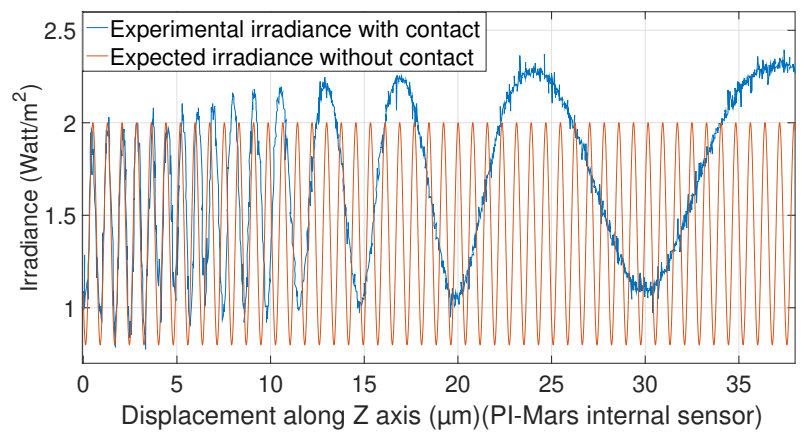

Fig. 11. Experimental FP interferences evolution when the photonic component is translated along the optical axis, a contact occurs at $Z=$ $6.16 \mu \mathrm{m}$.

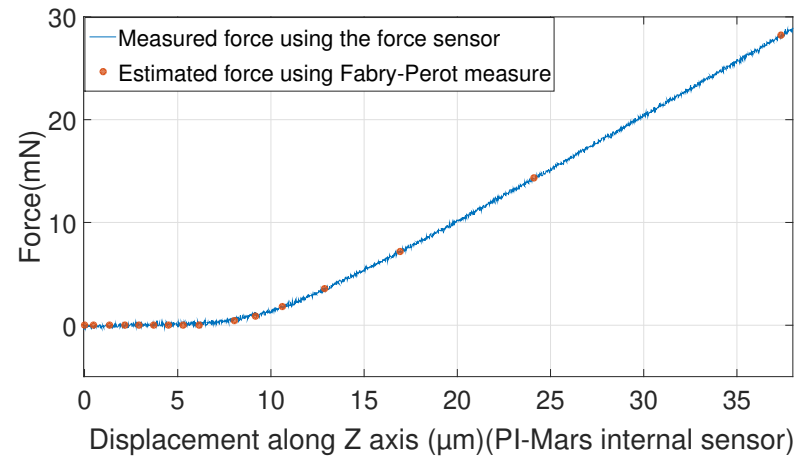

Fig. 12. Reconstruction of the contact force estimation based on FP interferences.

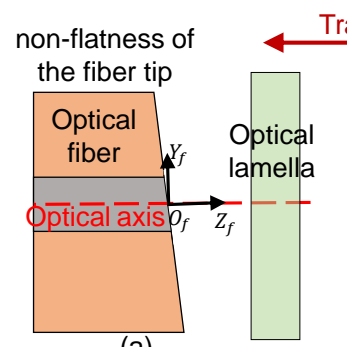

(a)
Translation direction of Optical lamella the optical lamella motion after contact

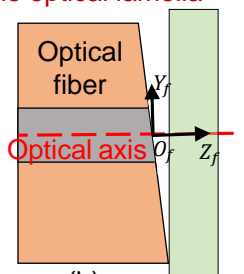

(b)

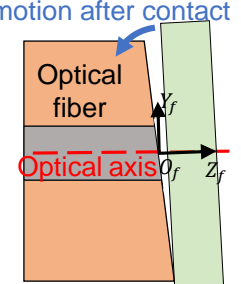

(c)
Fig. 13. (a) Optical lamella perpendicular to the optical axis (no contact), (b) Contact detection with zero force, (c) Contact with applied force (force estimation).

Translating the optical lamella toward the optical lamella produces simultaneously a periodic sinusoidal irradiance

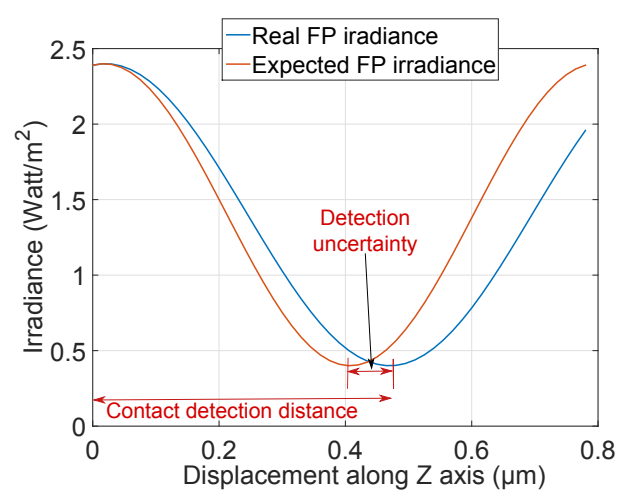

Fig. 14. Contact detection from expected and real FP irradiance periodicity difference.

with variable frequency as shown in Figure 11. Before contact detection between the optical lamella and the fiber tip, it represents the case of Figure 13 (a), which produces a signal of the period of $780 \mathrm{~nm}$, which corresponds to half of the used $1560 \mathrm{~nm}$ laser wavelength. At $z=6.16 \mu \mathrm{m}$, the contact occurs between the optical lamella and the fiber, which represents the case of Figure 13 (b). After contact detection, it represents the case of Figure 13 (c), an applied force by the optical lamella, it conducts to frequency reduction of the measured signal. This frequency reduction is due to the non-flatness of the fiber (fabrication defects), which is not considered in the photo-robotic approach for multi-DOF nanopositioning of the optical lamella with respect to the fiber in section IV.

The difference between this measured signal and the one when no contact exists (red curve named expected irradiance without contact) enables the estimation of the compliant structure compression. Knowing the stiffness of the compliant structure $(k=1.15 \mathrm{mN} / \mu \mathrm{m})$, it could also be possible to estimate the applied force. The estimated force can be calculated using equation 2 .

$$
F_{e}=k\left(X_{e}-X_{r}\right),
$$

The expected displacement $X_{e}$ before contact can be calculated using the same method as presented in section II or it can be taken from the internal sensor of the nanopostioner. After contact, the number of the produced peaks $p$ due to each movement are calculated. Then, the real displacement $X_{r}$ can be calculated using the same formula as presented in section II : $X_{r}=p * \lambda / 2$.

In order to validate the force estimation after contact based on FP interferometry, it is compared with the force measured by the reference sensor as shown in Figure 12 Results highlight a very good correspondence between both curves. They also show that it is possible to estimate the location of the contact with high precision. These results validate the proposed approach and clearly state the interest for force measurement based on the optical FP interferometry principle.

When the contact occurs, the frequency of the measured irradiance reduces. The difference between this measured irradiance and the one when no contact occurs (red curve 


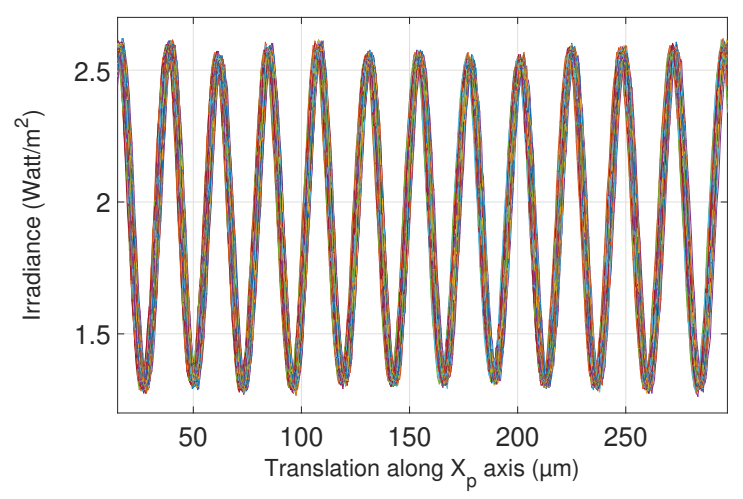

Fig. 15. Repeatability of the reflected light irradiance during experimentation for 30 translations.

named expected irradiance without contact) enables the contact detection as shown in Figure 14. The corresponding flowchart for contact detection using FP interferometry irradiance measurement is shown in Figure 16 By taking the case when there is no contact, the experimental optical irradiance uncertainty of Figure 15 corresponds to a $27.6 \mathrm{~nm}$ measurement uncertainty of displacement. When there is a contact the frequency of oscillation of the optical irradiance gets slower. By fixing a periodicity threshold more than the measurement uncertainty, hence, if the optical irradiance periodicity is more than the periodicity threshold, then there is a contact detection. This threshold is fixed to $80 \mathrm{~nm}$.

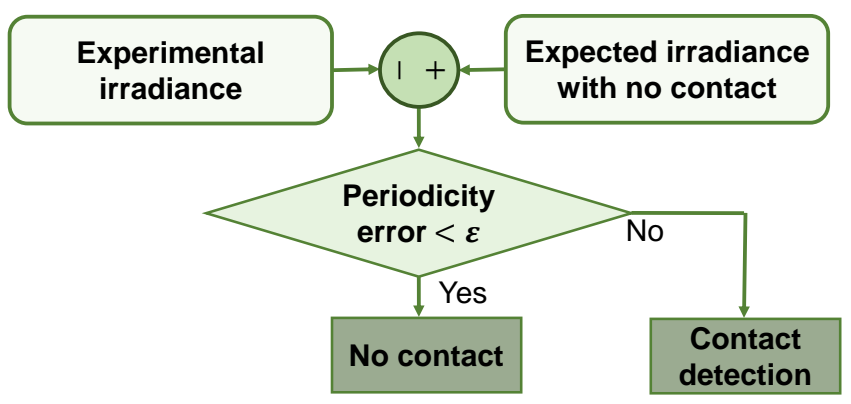

Fig. 16. Flowchart for contact detection based on FP interferometry measure.

\section{CONCLUSION}

In conventional active alignment, the non-referenced motion of the nano-positioner induces difficulties to analyse the source of this FP cavity variation, which leads to three main drawbacks: time consumption especially for multiDOF alignment process to reach maximum irradiance, local maximum problem and the alignment task success relies on human expertise.

A "position"-based photo-robotic positioning approach has been used for high accurate multi-DOF positioning of an optical lamella with respect to a fiber in free space. It relies on multi-DOF robot motions associated with 1-D FP interferometry measurements, to estimate poses in a multiDOF way.
An original contact detection approach has been proposed conducting to an original "force-position"-based photorobotic approach. It relies on correlation between the interference figure and the expected irradiance deduced from the nano-positioner internal sensors. The contact detection enables to estimate the position of the lamella with respect to the fiber along the optical axis which is, for example, usefull to control the UV-adhesive thickness thus achieving high optical performances after completing the assembly process.

Rotational measurements are achieved with an uncertainty based on standard deviation of $2.1 \mathrm{~m}^{\circ}$, and translational measurements with an uncertainty based on standard deviation of $27.6 \mathrm{~nm}$. Additionally, in constrained space where contact detection is required, the proposed contact detection is based on the correlation between the interference figure and the expected irradiance deduced from the nano-positioner internal sensors. This photo-robotic approach has especially been used to achieve automated multi-DOF assembly of an optical lamella relative to an optical fiber with high accuracy. Experimental results prove the high accurate contact detection with an error of $80 \mathrm{~nm}$ as a maximum.

\section{ACKNOWLEDGMENT}

These works have been funded by the Franche-Comté region, nano-roboptic project supported by the EUR EIPHI Graduate School (ANR-17-EURE-0002), ANR CEPAGE ANR-16-CE24-0024 and by the French RENATECH network through its FEMTO-ST technological facility.

\section{REFERENCES}

[1] C. Clévy, I. Lungu, K. Rabenorosoa, and P. Lutz, "Positioning accuracy characterization of assembled microscale components for micro-optical benches," Assembly Automation, vol. 34, no. 1, pp. 69$77,2014$.

[2] D. O. Popa, R. Murthy, and A. N. Das, " $\mathrm{M}^{3}$-deterministic, multiscale, multirobot platform for microsystems packaging: Design and quasistatic precision evaluation," IEEE Transactions on Automation Science and Engineering, vol. 6, no. 2, pp. 345-361, 2009.

[3] A. N. Das, R. Murthy, D. O. Popa, and H. E. Stephanou, "A multiscale assembly and packaging system for manufacturing of complex micro-nano devices," IEEE Transactions on Automation Science and Engineering, vol. 9, no. 1, pp. 160-170, 2012.

[4] O. Benson, "Assembly of hybrid photonic architectures from nanophotonic constituents," Nature, vol. 480, no. 7376, pp. 193-199, 2011.

[5] G. Böttger, D. Weber, F. Scholz, H. Schröder, M. Schneider-Ramelow, and K.-D. Lang, "Fully automated hybrid diode laser assembly using high precision active alignment," in Components and Packaging for Laser Systems II, vol. 9730, p. 97300E. International Society for Optics and Photonics, 2016.

[6] R. Hauffe, U. Siebel, K. Petermann, R. Moosburger, J.-R. Kropp, and F. Arndt, "Methods for passive fiber chip coupling of integrated optical devices," IEEE transactions on advanced packaging, vol. 24, no. 4, pp. 450-455, 2001.

[7] R. Takigawa, E. Higurashi, T. Suga, and T. Kawanishi, "Passive alignment and mounting of linbo _3 waveguide chips on si substrates by low-temperature solid-state bonding of au," IEEE Journal of Selected Topics in Quantum Electronics, vol. 17, no. 3, pp. 652-658, 2011.

[8] Y. Ma, X. Liu, J. Zhang, D. Xu, D. Zhang, and W. Wu, "Robotic grasping and alignment for small size components assembly based on visual servoing," The International Journal of Advanced Manufacturing Technology, vol. 106, no. 11, pp. 4827-4843, 2020.

[9] Y. Zheng, X.-c. Kai, J.-a. Duan, and B.-b. Li, "Automated visual position detection and adjustment for optical waveguide chips and optical fiber arrays," Journal of Central South University, vol. 22, pp. 3868-3875, 2015. 
[10] R. Zhang and F. G. Shi, "A novel algorithm for fiber-optic alignment automation," Advanced Packaging, IEEE Transactions on, vol. 27, no. 1, pp. 173-178, 2004.

[11] M. Landry, A. Kaddouri, Y. Bouslimani, and M. Ghribi, "Automated alignment of microstructured optical fibers and conventional singlemode fibers," Optical Engineering, vol. 52, no. 6, p. 065003, 2013.

[12] N. Tan, C. Clévy, G. J. Laurent, P. Sandoz, and N. Chaillet, "Accuracy quantification and improvement of serial micropositioning robots for in-plane motions," IEEE Transactions on robotics, vol. 31, no. 6, pp. 1497-1507, 2015.

[13] V. Guelpa, A. V. Kudryavtsev, N. L.-F. Piat, and S. Dembélé, "Accurate 3d-positioning in a sem through robot calibration," in IEEE International Conference on Manipulation, Automation and Robotics at Small Scales, pp. 1-6, 2018.

[14] H. Bettahar, A. Caspar, C. Clévy, N. Courjal, and P. Lutz, "Photorobotic positioning for integrated optics," IEEE Robotics and Automation Letters, vol. 2, no. 1, pp. 217-222, 2016.

[15] H. Bettahar, C. Clévy, F. Behague, N. Courjal, and P. Lutz, "Novel strategy for high precision automated robotic positioning based on fabry-perot interferometry principle," in IEEE 14th International Conference on Automation Science and Engineering, pp.1441-1447, 2018.

[16] H. Bettahar, O. Lehmann, C. Clévy, N. Courjal, and P. Lutz, "Photorobotic extrinsic parameters calibration of 6-dof robot for high positioning accuracy," IEEE/ASME Transactions on Mechatronics, vol. 25, no. 2, pp. 616-626, 2020 